\section{ORIGINAL RESEARCH}

\author{
A. Dinçer \\ S. Kohan \\ M.M. Özek
}

\title{
Is All "Communicating" Hydrocephalus Really Communicating? Prospective Study on the Value of 3D-Constructive Interference in Steady State Sequence at 3T
}

\begin{abstract}
BACKGROUND AND PURPOSE: 3D-constructive interference in steady state (3D-CISS) sequence has been used to assess the CSF pathways. The aim of this study was to investigate the additive value of 3D-CISS compared with conventional sequences in the diagnosis of obstructive membranes in hydrocephalus.
\end{abstract}

\begin{abstract}
MATERIALS AND METHODS: A total of 134 patients with hydrocephalus underwent MR imaging examination with a $3 \mathrm{~T}$ unit consisting of turbo spin-echo, 3D-CISS, and cine phase-contrast (cine PC) sequences. 3D-CISS was used to assess obstructive membranes in CSF pathways compared with other sequences. Cine PC, follow-up imaging, and surgical findings were used to confirm obstructive membranes.
\end{abstract}

\begin{abstract}
RESULTS: Comparing the number of noncommunicating cases by using the conventional and 3D-CISS images, we found 26 new cases $(19.4 \%)$ of 134 cases that were previously misdiagnosed as communicating hydrocephalus by conventional images. 3D-CISS sequence identified obstructive membranes invisible in other sequences, which facilitated selection of neuroendoscopy in the treatment of 31 patients $(23.1 \%)$ in total who would have been otherwise treated with shunt insertion. These patients included 26 newly diagnosed noncommunicating cases after demonstration of intraventricular and/or fourth ventricular outlet membranes and 5 cases of communicating hydrocephalus with obstructing cisternal membranes. There were obstructions of the foramina of Luschka in 22 of 26 newly found noncommunicating cases.
\end{abstract}

CONCLUSIONS: Conventional sequences are insensitive to obstructive membranes in CSF pathways, especially in the fourth ventricular exit foramina and the basal cisterns. 3D-CISS sequence, revealing these obstructive membranes, can alter patient treatment and prognosis.

$3^{\mathrm{D}}$ D-constructive interference in the steady state (3D-CISS) is fully balanced and inherently flow-compensated gradient-echo sequence providing fine anatomic details about CSF pathways. ${ }^{1}$ Two true-fast imaging with steady-state precession sequences with differing radio-frequency pulses are combined for strong T2/T1-weighted high-resolution 3D images. ${ }^{1-4}$ The advantage of the 3D-CISS sequence is its combination of high signal intensity levels and extremely high spatial resolution. During the past decade, 3D-CISS sequence has been introduced and used in the assessment of normal and pathologic features of intracranial anatomy. ${ }^{1-11}$ In particular, the value of this technique has been demonstrated in hydrocephalus and endoscopic third ventriculostomy. ${ }^{12-17}$ The T2-weighted turbo spin-echo (TSE) sequence has commonly been used to study the CSF pathway in hydrocephalus; however, it fails to demonstrate thin membranes causing obstruction at various foraminal levels and cisterns. ${ }^{15}$ From a treatment point of

Received March 23, 2009; accepted after revision May 4.

From Acibadem University, School of Medicine, Departments of Radiology (A.D.) and Neurosurgery (M.M.Ö.), Istanbul, Turkey; University of New South Wales (S.K.), St. George Hospital, Sydney, Australia; and Marmara University Medical Center, Division of Pediatric Neurosurgery (M.M.Ö.), Istanbul, Turkey.

Paper previously presented at: American Society of Neuroradiology Annual Meeting and Neuroradiology Education and Resarch Foundation Symposium, New Orleans, La, June 5 , 2008.

Please address correspondence to Alp Dinçer, MD, Acibadem Kozyatagi Hospital, Department of Radiology, Inonu Cad. Okur Sok. No:21, Kozyatagı, Istanbul, 34742 Turkey; e-mail: adincer@asg.com.tr or alpdincer@yahoo.com

DOI 10.3174/ajnr.A1726 view, identification of obstructive pathologic processes at any level through the CSF pathway is of significant importance because it can change the mode of management in patients with hydrocephalus, avoiding shunt insertion.

In this prospective study, we aimed to investigate the additive value of $3 \mathrm{D}$-CISS sequence compared with conventional T1-weighted and T2-weighted images at $3 \mathrm{~T}$ in the diagnosis of obstructive membranes in patients who would otherwise be diagnosed with communicating hydrocephalus.

\section{Materials and Methods}

\section{Patients}

A total of 134 consecutive patients, 84 male and 50 female, mean age 10.3 years (range, 2 days to 18 years) with hydrocephalus who were evaluated in our MR imaging unit between January 2005 and February 2009 were included in the study. All patients were examined with a 3T MR imaging machine with an 8-channel head coil (Trio; Siemens, Erlangen, Germany). Our hospital internal review board approved the study. All patients or caregivers were informed about the study and had given consent for participation. All patients younger than 10 years were examined routinely under sedation.

\section{MR Imaging Technique}

All patients had completed conventional study including axial TSE T1, and axial, sagittal, and coronal TSE T2, sagittal 3D turbo fast low angle shot (turboFLASH) T1, and sagittal 3D-CISS followed by sagittal and axial-oblique cine phase-contrast (cine PC). The 3D-CISS sequence was acquired with $0.6 \times 0.6 \times 0.6-\mathrm{mm}^{3}$ isometric voxel 


\begin{tabular}{|c|c|c|c|c|c|c|}
\hline Sequences & $\begin{array}{l}\text { Imaging } \\
\text { Plane }\end{array}$ & TR/TE/ETL & $\begin{array}{c}\text { Time of } \\
\text { Acquisition }\end{array}$ & $\begin{array}{l}\text { Voxel Size } \\
\left(\mathrm{mm}^{3}\right)\end{array}$ & $\begin{array}{c}\text { Section/Partition } \\
\text { Thickness/Gap (mm) }\end{array}$ & $\begin{array}{l}\mathrm{FA} / \mathrm{BW} \\
(\mathrm{Hz} / \mathrm{Px})\end{array}$ \\
\hline TSE T2 & Axial & $3590 / 101 / 13$ & 1.53 & 1.75 & $5 / 0.5$ & $90 / 100$ \\
\hline TSE T2 & Sagittal & $3000 / 131 / 13$ & 1.41 & 1.2 & $2 / 0.2$ & $90 / 100$ \\
\hline TSE T2 & Coronal & 4850/132/13 & 1.36 & 1.08 & $3 / 0.75$ & $90 / 100$ \\
\hline Cine PC & Oblique & $59 / 11 /-$ & $3-5$ & 1.08 & 3 & $35 / 391$ \\
\hline 3D Turbo flash T1 & Sagittal & $2000 / 3.9 /-$ & 4.14 & 0.51 & 0.8 & $20 / 130$ \\
\hline TSE T1 & Axial & $500 / 13 / 2$ & 2 & 2.05 & $5 / 0.5$ & $70 / 155$ \\
\hline 3D-CISS & Sagittal & $13.6 / 5.7 /-$ & 4.44 & 0.216 & 0.6 & $50 / 130$ \\
\hline
\end{tabular}

Note:-ETL indicates echo-train length; FA, flip angle; BW, bandwidth; TSE, turbo spin-echo; cine PC, cine phase-contrast; 3D turbo flash T1, 3D turbo fast low-angle shot T1; 3D-CISS, $3 \mathrm{D}$ constructive interference in the steady state.

allowing reconstruction in any arbitrary plane for optimal visualization. The various sequence parameters are summarized in Table 1 . If needed, axial TSE T1 and sagittal 3D turboFLASH T1 were repeated after contrast administration. The total scan time for noncontrast examination was approximately 25 minutes depending on the time required for cine PC. All sequences including 3D-CISS sequence and cine $\mathrm{PC}$ were obtained at the same session for all patients.

3D-CISS sequence was applied in the sagittal plane to cover the entire ventricular region and the fourth ventricular exits with the following parameters: TR, $10.94 \mathrm{~ms}$; TE, $5.47 \mathrm{~ms}$; flip angle (FA), $50^{\circ}$; FOV $200 \times 180 \mathrm{~mm}$; matrix, $320 \times 320$; bandwidth, $130 \mathrm{~Hz} /$ pixels; partition/slab thickness, $0.6 / 28.8 \mathrm{~mm}$; partition number, 48 ; section oversampling, $11 \%$; phase oversampling, $25 \%$; and measurement, 1. The 3D-CISS sequence was located at the midline. If needed, the partition number was increased up to 96 .

Midline high-resolution sagittal cine PC was obtained for all patients as a standard sequence. The sequence parameters were as follows: TR, $37 \mathrm{~ms}$; TE, $5.6 \mathrm{~ms}$; averages, 3; FOV, $216 \times 240 \mathrm{~mm}$; section thickness, $2 \mathrm{~mm}$; FA, $30^{\circ}$; matrix, $256 \times 256$; voxel size, $0.9 \times$ $0.9 \times 2 \mathrm{~mm}^{3}$; velocity encoding, $20 \mathrm{~cm} / \mathrm{sn}$; direction of flow encoding, foot to head; and bandwidth, $391 \mathrm{~Hz} /$ pixels. Electrocardiogram or pulse trigger was used with a $90 \%$ acquisition window. Just after acquisition, magnitude images, rephrased, and phase images were reconstructed immediately. According to the anatomic region, the axial-oblique or sagittal-oblique cine PC was obtained with the same parameters except for the velocity encoding direction, which was set to the through plane. Cine PC was evaluated with vendor-supplied, commercially available software (Argus on a Leonardo workstation; Siemens, Erlangen, Germany).

\section{MR Evaluation}

Our diagnostic criterion for obstructive membranes depended on demonstration of the membrane itself by both conventional T1weighted and $\mathrm{T} 2$-weighted images and/or by $3 \mathrm{D}$-CISS sequence. Only membranes that could be followed all the way and divided the anatomic region completely into 2 or more parts were accepted as obstructive membranes. The nonobstructive membranes, which are normal cisternal structures, have been known for more than 100 years. ${ }^{2,18,19}$ An increased number of such normal cisternal structures such as the Liliequist membrane could be visible with high-resolution cisternal imaging, and these normal structures were not taken into consideration as obstructive membranes. Signal intensity differences were not treated as a primary finding for making a decision as to the existence of an obstructive membrane. A sharp, linear zone of a transition signal intensity difference between 2 sides of the obstructive membrane was considered as only a complementary finding.

Every examination was performed step by step in the following order under close supervision of an experienced radiologist (A.D, 16 years' experience in neuroradiology). First, routine sequences were obtained. During 3D-CISS sequence imaging, a judgment was made if an obstructive pathologic feature existed, and the diagnosis of communicating or noncommunicating hydrocephalus was made by the radiologist on the basis of conventional T1-weighted and T2weighted images. During the first step, every effort was made by the radiologist to detect the presence of any obstructive membrane on the basis of routine imaging. The Dandy traditional classification of hydrocephalus was used. ${ }^{20,21}$ This classification system depends on administering a dye into the lateral ventricle and then trying to detect the dye in the CSF sample collected by lumbar puncture. On the basis of this definition, hydrocephalus cases with obstructive cisternal membrane only, without accompanying intraventricular or fourth ventricular exit foramina obstruction, were defined as a communicating hydrocephalus. The membranous obstruction at the foramen of Monro, the cerebral aqueduct, foramen of Magendie, foramina of Luschka, and superior medullary velum were defined as intraventricular membranous obstruction. The membranes that obscured only the cisterns (ie, interpeduncular, prepontine, and premedullary) were defined as communicating hydrocephalus with cisternal obstruction. ${ }^{22-24}$ The hydrocephalus was categorized as noncommunicating, even if one of the fourth ventricular outlets appeared obstructed while the others were patent. Although this assumption seems to be against traditional concept, a few case reports have been published with only 1 exit foramen of the fourth ventricle obstructed, causing tetraventricular hydrocephalus and responding to surgery. ${ }^{23-32}$ These results overlap with observation in our large study cohort.

The second step was the acquisition of the 3D-CISS sequence to determine whether there was obstructing membrane either within or outside the ventricular system. The 3D-CISS images were examined during acquisition of the standard sagittal cine PC sequence with in-plane flow sensitization gradient. We carefully scrutinized every section and used vendor-provided 3D multiplanar reconstruction software that allows us real-time multi-planar postprocessing.

For the final step, cine PC images were used to confirm the obstructive nature of the pathologic condition. Cine PC was considered to be the criterion standard. For consideration of a cisternal or ventricular membrane seen on 3D-CISS sequence as obstructive, no clear in-plane or through-plane flow had to be demonstrated across the membrane. Every possible region with obstructive membrane was studied carefully with cine PC. Midline sagittal cine PC with in-plane flow-sensitization gradient was obtained for all patients. According to the location of the membrane, axial-oblique or sagittal-oblique cine PC with through-plane sensitization gradient was obtained perpendicular to the cerebral aqueduct at the narrowest portion for aqueduct stenosis, below the aqueduct for superior velum medullary synechia, at the fourth ventricle floor for the foramen of Magendie, parasagittal- 


\begin{tabular}{lcccccccc}
\hline \multicolumn{1}{l}{ Table 2: Site of membranous obstruction on the basis of examination of different sequences } \\
\hline
\end{tabular}

* These cases were indirectly diagnosed on the basis of unilateral or bilateral lateral ventricular dilation. There are 2 bilateral membranes in 5 cases.

Table 3: Classification of hydrocephalus on the basis of examination of different sequences

\begin{tabular}{|c|c|c|c|c|c|}
\hline \multirow[b]{2}{*}{ Sequences } & \multicolumn{2}{|c|}{$\begin{array}{c}\text { Number of Communicating } \\
\text { Hydrocephalus Cases }\end{array}$} & \multicolumn{2}{|c|}{$\begin{array}{c}\text { Number of Noncommunicating } \\
\text { Hydrocephalus Cases with } \\
\text { Obstructive Membranes }\end{array}$} & \multirow[b]{2}{*}{$\begin{array}{c}\text { Total Number } \\
\text { of Cases with } \\
\text { Obstructive } \\
\text { Membranes }\end{array}$} \\
\hline & $\begin{array}{l}\text { Number of Cases } \\
\text { with no Visible } \\
\text { Obstructive } \\
\text { Membrane }\end{array}$ & $\begin{array}{c}\text { Number of Cases } \\
\text { with Only an } \\
\text { Obstructive } \\
\text { Cisternal } \\
\text { Membrane }\end{array}$ & $\begin{array}{c}\text { Number of Cases } \\
\text { with Only an } \\
\text { Obstructive } \\
\text { Intraventricular } \\
\text { Membrane }\end{array}$ & $\begin{array}{l}\text { Number of Cases } \\
\text { with Obstructive } \\
\text { Ventricular Membrane } \\
\text { Plus Obstructive } \\
\text { Cisternal Membrane }\end{array}$ & \\
\hline Conventional T1 and T2 & 46 & 0 & 47 & 1 & 48 \\
\hline 3D-CISS & 13 & 7 & 63 & 22 & 92 \\
\hline
\end{tabular}

oblique for the foramina of Luschka and Monro, and perpendicular to the prepontine cistern for cisternal membrane obstruction.

Just after the examination, the radiologist and the neurosurgeon (M.M.Ö.) made a final decision by consensus to use all of the data together. Both the radiologist and the neurosurgeon knew all patient data available.

\section{Treatment of Patients}

According to the type of hydrocephalus, either endoscopic third ventriculostomy (ETV) was performed or a shunt was inserted. A total of 26 new cases were diagnosed as noncommunicating hydrocephalus with 3D-CISS and were treated with ETV. All patients were followed-up at 6-months intervals. ETV was used to confirm cisternal membranes, membranes of the foramen of Monro, and aqueduct stenosis. Clinical and MR imaging follow-up findings were used to confirm the obstruction at the superior velum medullary synechia, foramina of Luschka, foramen of Magendie, and foramen magnum alongside the initial cine PC findings.

\section{Results}

3D-CISS sequence demonstrated 157 sites of membranous obstruction in 134 patients. The location of these membranes were as follows: 7 at the foramen of Monro (bilateral in 2 cases), 41 at the cerebral aqueduct, 5 at the superior medullary velum, 28 at the foramen of Magendie, 46 at the foramina of Luschka, 2 at the foramen magnum, and 28 in the cisterns. Although conventional images showed only 57 sites of membranous obstruction in these 134 patients, the conventional images showed all aqueduct stenosis and membranous obstruction of the foramen of Monro, 1 of 5 superior medullary velum synechia, 4 of 28 foramen of Magendie membranes, 3 of 46 foramina of Luschka membranes, and 1 of 28 cisternal membranes. In the conventional T1 and T2 images, all membranes of the foramen of Monro were diagnosed with indirect findings such as unilateral or bilateral lateral ventricle dilation with a small third ventricle. Indeed, we did not demonstrate any membrane of the foramen of Monro itself with the conventional images. The sites of membranous obstruction are summarized in Table 2 .
On the basis of the conventional images, there were 46 cases $(34.3 \%)$ of communicating hydrocephalus and 88 cases $(65.7 \%)$ of noncommunicating hydrocephalus. Of the 88 noncommunicating cases, only 48 cases $(54.5 \%)$ were diagnosed with membranous obstruction (41 of the aqueduct stenosis, 5 membranous obstruction of the foramen of Monro, 1 obstruction of the superior medullary velum synechia, and 1 cisternal membranous obstruction). In 40 remaining cases (45.5\%) no obstructive membrane was identified (Table 3 ), and the diagnosis was made on the basis of indirect findings.

Examining 3D-CISS images demonstrated 20 cases $(14.9 \%)$ with communicating hydrocephalus and 114 cases (85.1\%) of noncommunicating hydrocephalus (Table 3 ). Comparing the number of noncommunicating cases by using the conventional and 3D-CISS images, we found 26 new cases (19.4\%) of 134 cases that were previously misdiagnosed as a communicating hydrocephalus on conventional images. In addition to these new 26 intraventricular membranous obstructions, we found another 7 previously undiagnosed cases with cisternal obstructive membranes, which were not classified as noncommunicating hydrocephalus according to the Dandy traditional concept. Furthermore, by using the 3DCISS sequence, we were able to demonstrate fine membranous obstructive structures within the CSF pathway in 92 cases $(68.7 \%)$ of the total number of cases examined. These 92 cases consisted of 33 cases with previously undiagnosed membranous structures (26 intraventricular and 7 cisternal obstructive membrane cases), 48 cases that were already identified on conventional images, and 11 with more than 1 obstructive pathologic feature (arachnoid cysts in 7 cases, tectal mass in 4 cases). On the basis of the 3D-CISS images, of the 114 cases of noncommunicating hydrocephalus, 85 had obstructive intraventricular membranes, 29 had space-occupying lesions, and 11 had both of these features.

A history of intracranial hemorrhage or infection was noted in 38 of a total number of 134 cases. Cisternal obstructive membranes were demonstrated with $3 \mathrm{D}$-CISS sequence in 28 of these cases. The cisternal localization of obstructive membranes in these patients was as follows: prepontine mem- 

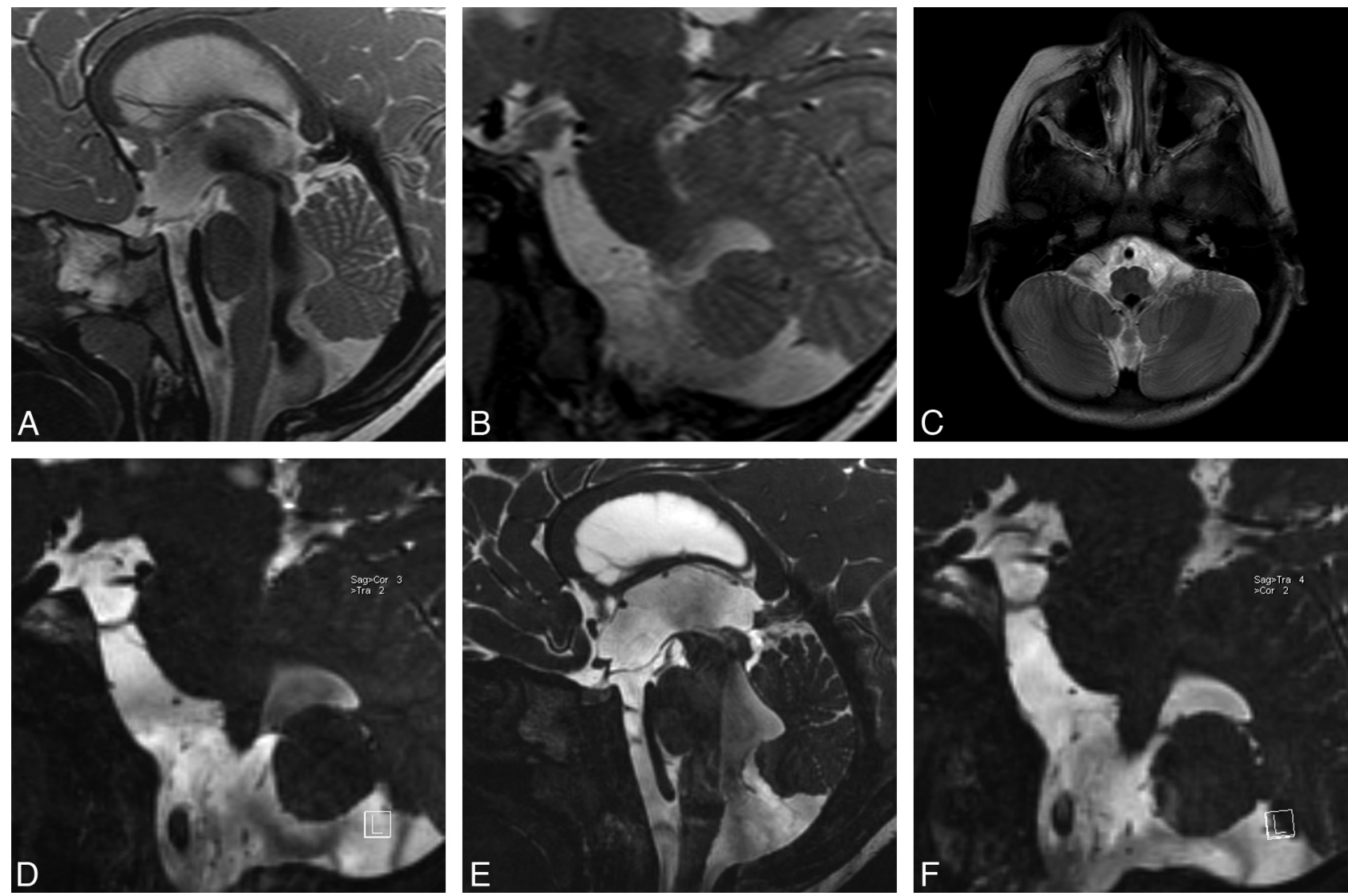

Fig 1. Case 1. A, Sagittal TSE T2 image shows third ventricle enlargement with downward displacement of the floor of the third ventricle consistent with hydrocephalus. The cerebral aqueduct and foramen of Magendie are open widely, showing extensive flow void phenomenon. There is a mild enlargement in the fourth ventricle. There is no sign of obstructive membrane in the prepontine cistern. $B$, Left parasagittal TSE T2 images through the left lateral ventricular exit show no direct or indirect sign of membrane. C, Axial TSE T2 image through the fourth ventricular exits demonstrates prominent signal intensity void in the fourth ventricle, but there is no direct or indirect sign of obstructive membrane at the foramina of Luschka. $D$, Left parasagittal 3D-CISS image clearly points out the membrane itself in the foramen of Luschka. There is extensive intensity difference between the fourth ventricle and the neighboring cistern. E, Sagittal 3D-CISS image clearly demonstrates prepontine membranes extending from the clivus to the basilar artery. F, Left parasagittal follow-up 3D-CISS image indicates persistent membrane in the fourth ventricle exit foramen, though the intensity differences between cistern and ventricle have disappeared.

branes only in 9 patients, interpeduncular cisternal membranes only in 4 patients, prepontine and interpeduncular cisternal membranes in 11 patients, and extensive obstructive cisternal membrane formation in 4 patients. The membranes in patients without a history of infection or hemorrhage were defined as congenital if the membranes were thin and restricted to a maximum of 2 ventricular sites. Of the 92 cases with membranous obstruction, 24 cases had congenital membranes.

Seventeen of 28 cases with cisternal membranous obstructions were proved surgically during the ETV procedures. Four of 5 membranes at the foramen of Monro and 33 of 41 aqueduct stenoses were demonstrated and treated endoscopically. All cases with superior velum medullary synechia were treated with ETV. All cases with foramen of Magendie, foramen of Luschka, and foramen magnum membranes were treated either with ETV or shunt insertion. Posterior fossa surgery was not performed in these cases.

The location of the membranes in 26 new cases diagnosed only with 3D-CISS sequence as a noncommunicating hydrocephalus were as follows: membranes of the foramen of Lusch$\mathrm{ka}$ in 6 cases; membranes of the foramen of Magendie in 2 cases; superior medullary velum synechia in 4 cases; membranes of the foramina of Luschka and cisterns in 8 cases; and membranes of the foramina of Luschka, Magendie, and cis- terns in 6 cases. In these cases, ETV was performed. Patients were followed up for 6 months to 3 years. During follow-up, stomal closure was diagnosed in 4 cases. A second ETV procedure was attempted in 3 of them. After the second attempt, 1 patient remained well during the 1 year follow-up and the other 3 patients were treated with shunt insertion. The overall success rate of ETV was $88.5 \%$ (23/26) in this group, with up to 3 years of follow-up.

\section{Case Illustrations}

Case 1. A 3-year-old girl was admitted to the hospital with vomiting. Neurologic examination showed increase in head size. Conventional T1 and T2 images demonstrated bilateral lateral ventricular dilation. A sagittal TSE T2 image showed an enlarged third ventricle with extensive downward displacement of the floor of the third ventricle. The cerebral aqueduct was open, and there was mild enlargement of the fourth ventricle, with both demonstrating extensive flow void phenomenon, compatible with flow (Fig 1A). There was no sign of obstructive membranes in TSE T2 images in the parasagittal and axial planes (Fig $1 B$ and $1 C$ ). However, parasagittal oblique reformatted images of the 3D-CISS sequence clearly demonstrated membranes in the foramina of Luschka, which obstructed the fourth ventricular exits bilaterally (Fig $1 D$ ). Sagittal 3D-CISS images also revealed the prepontine mem- 

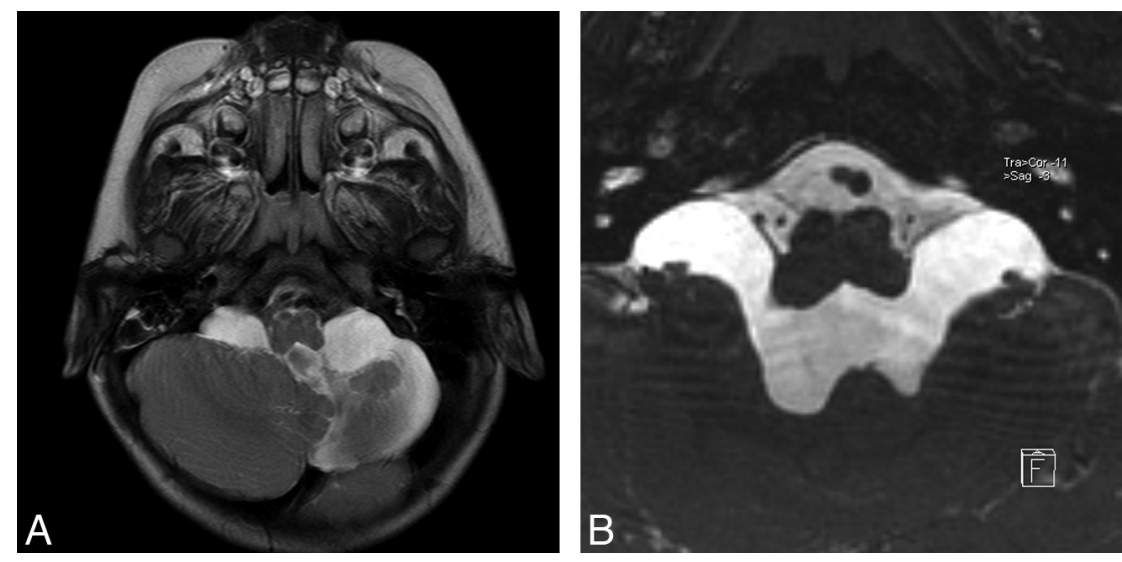

Fig 2. Case 2. A, Axial TSE T2 image through the posterior fossa shows left cerebellar hypoplasia and enlargement of the bilateral cerebellomedullary cistern without any evidence of membrane at the fourth ventricle exit foramina. B, Axial oblique reformatted image of sagittal 3D-CISS reveals obstructing membranes of foramina of Luschka, bulging into the cerebellomedullary cisterns.
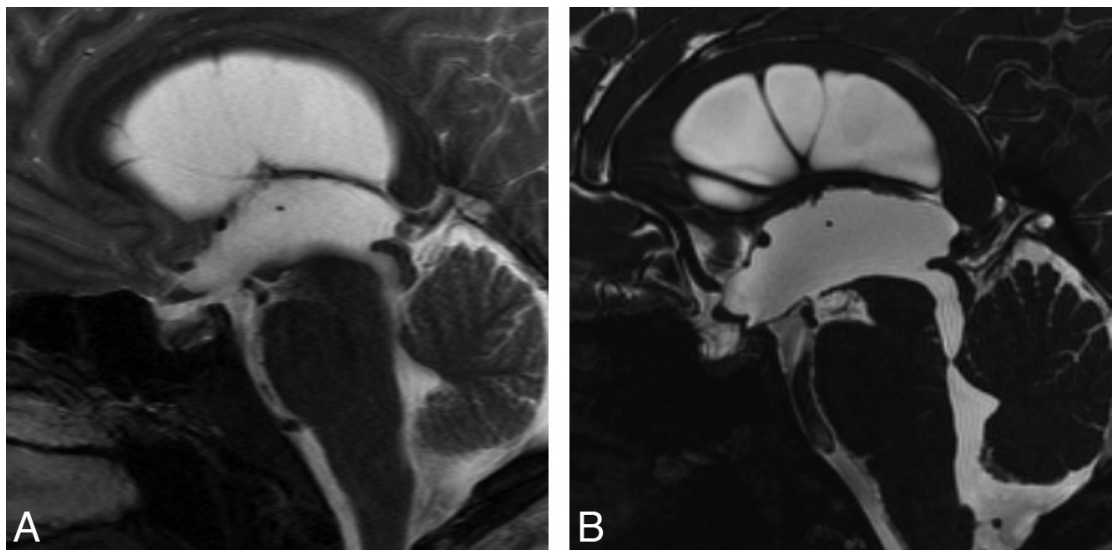

Fig 3. Case 3. A, Sagittal TSE T2 image shows an enlarged third ventricle. The cerebral aqueduct seems to be open. B, Sagittal 3D-CISS demonstrates superior medullary velum synechia causing triventricular hydrocephalus. There is a spontaneous third ventriculostomy at the floor of the ventricle, just behind the tip of the basilar artery. Sagittal CISS revealed the anatomic defect, whereas cine PC did not detect any flow through the defect.

brane extending from the clivus to the pons, which was again not seen in conventional images (Fig $1 E$ ). During the ETV procedure, the floor of the third ventricle and prepontine membrane were dilated with a balloon after perforation with blunt dissection. The 6-month follow-up MR imaging demonstrated diminished ventricular size with functional stoma. The parasagittal 3D-CISS reformatted images at 6 months showed a similar appearance of the foramina of Luschka membranes but disappearance of flow void in the fourth ventricle compared with the preoperative images (Fig $1 F$ ). The patient remained well after 3 years of follow-up.

Case 2. A 17-month-old girl was admitted to the hospital with weakness of the right hand and foot. She had a history of perinatal intracranial hemorrhage. Neurologic examination revealed right hemiparesis. Conventional images demonstrated asymmetric lateral ventricular dilation, more prominent on the left; sequel left basal ganglia lesion; and left cerebellar hypoplasia (Fig 2A). Sagittal images showed an enlarged third ventricle with extensive downward displacement of the ventricular floor. The cerebral aqueduct was open, and there was mild enlargement of the fourth ventricle, with both demonstrating extensive flow void phenomenon. There was no evidence of membrane at the foramen of Luschka on the conventional images (Fig 2A), whereas axial-oblique reformatted images of 3D-CISS sequence clearly demonstrated an outwardly bulging membrane that obstructed the fourth ventricular exits bilaterally (Fig 2B). The 6-month follow-up MR imaging after ETV demonstrated diminished ventricular size. The patient was well after 1-year follow-up with mild right hemiparesis.

Case 3. An 18-year-old man was admitted to the hospital with headache. Results of neurologic examination were unremarkable. Conventional images demonstrated mild triventricular hydrocephalus. There was no flow void in the cerebral aqueduct, but it appeared to be open in conventional T1 and T2-weighted images (Fig 3A). Sagittal 3D-CISS image demonstrated superior medullary velum synechia below the aqueduct (Fig 3B). Cine PC confirmed total occlusion at this region. ETV was performed and follow-up MR imaging at 6 months showed reduction in ventricular size.

Case 4. A 7-year-old girl was admitted to the hospital with transient double vision and headache. Results of neurologic examination were unremarkable at admission. Conventional images demonstrated moderate tetraventricular communicating hydrocephalus without evidence of cisternal or intraventricular membranes (Fig 4A), whereas the 3D-CISS sequence demonstrated prepontine membranes under the Liliequist membrane, extending from the clivus to the basilar artery. 

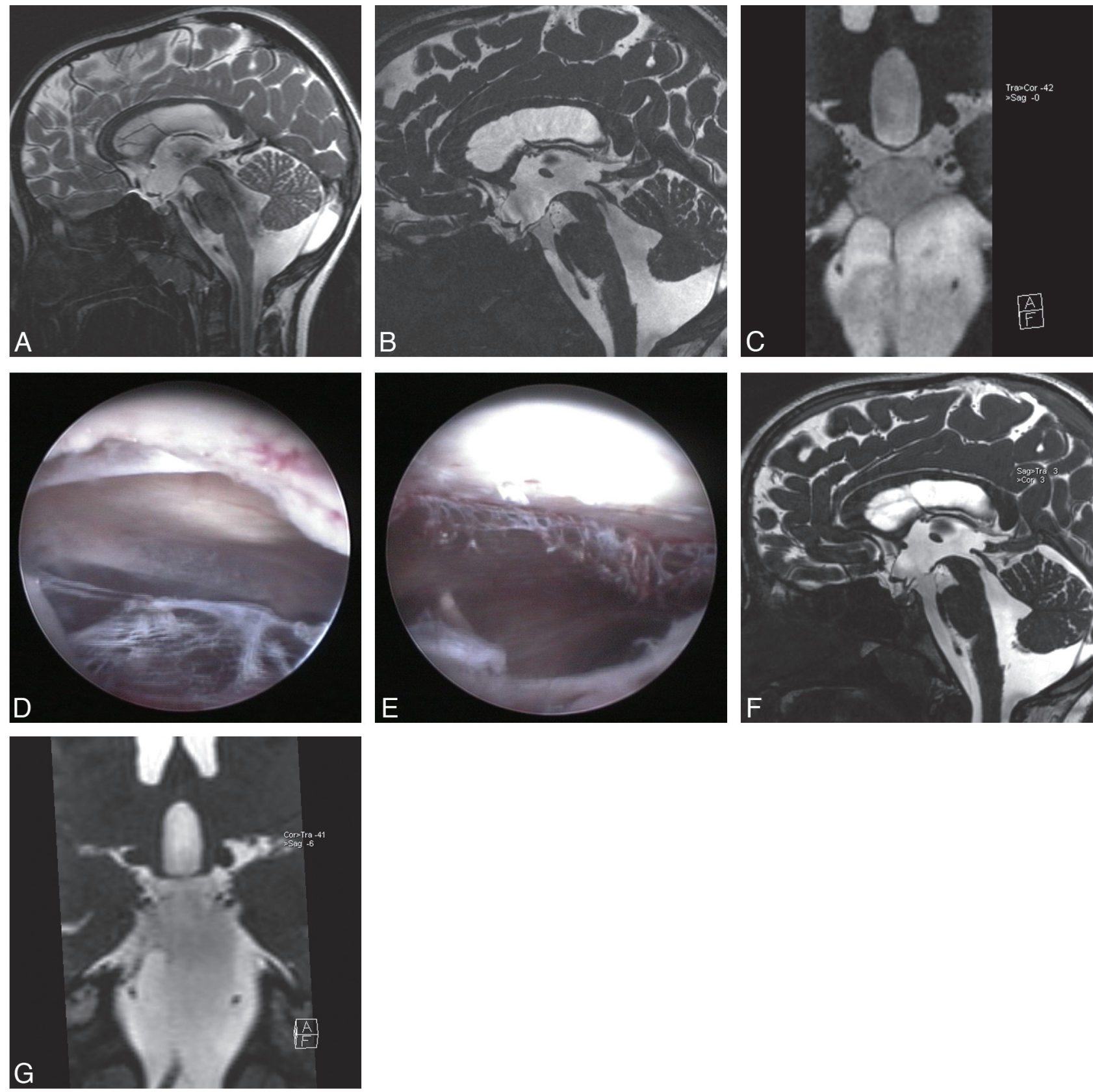

Fig 4. Case 4. A, Sagittal TSE T2 image demonstrates enlarged third ventricle, extensive flow void phenomenon in the cerebral aqueduct, the fourth ventricle, and prepontine/interpeduncular cisterns compatible with communicating hydrocephalus. B, Sagittal 3D-CISS image shows Liliequist membrane just below the downward bulging floor of the third ventricle. In addition to this, there is a thin membrane extending from the clivus to the basilar artery, dividing the prepontine cistern into upper and lower parts. The signal intensity difference between 2 parts with a sharp, linear zone of transition was seen. C. Coronal oblique reformatted image of 3D-CISS through the cisterns demonstrates lateral extension of the prepontine membrane, trapping CSF between the Liliequist membrane and the prepontine membrane. D, During ETV, after the floor of the third ventricle through the Liliequist membrane is opened, a complete membrane without any aperture is seen. E, The same view after the membrane is opened and removed, the cisternal part of the left abducens nerve is seen partly. F, On the follow-up sagittal 3D-CISS image, there is free communication between the third ventricle and the prepontine cistern through the interpeduncular cistern. G, Follow-up coronal oblique reformatted image of 3D-CISS after ETV through the cisterns (same view as in C) reveals nearly complete removal of the prepontine membrane. The difference in signal intensity between the 2 parts of the cisterns has almost completely disappeared.

There was clear a difference in intensity between the interpeduncular and prepontine cisterns with 3D-CISS images (Fig $4 B,-C)$. Hydrocephalus was classified as a communicating one with obstructive cisternal membranes. During the ETV, all membranes defined with 3D-CISS sequence were visualized and fenestrated (Fig 4D, -E). The patient was well without any complaint at 6-month follow-up, and follow-up MR imaging with 3D-CISS sequence demonstrated reduction in size of lat- eral ventricles and free communication between the third ventricle and prepontine cistern (Fig $4 F,-G$ ).

Case 5. A 2-month-old girl was admitted to the hospital with vomiting. Results of neurologic examination revealed bulging fontanelles and increased head circumference. TSE T2 images demonstrated right lateral ventricular enlargement without direct evidence of membranous obstruction of the foramen of Monro (Fig $5 A,-B$ ). Coronal reformatted 3D-CISS 

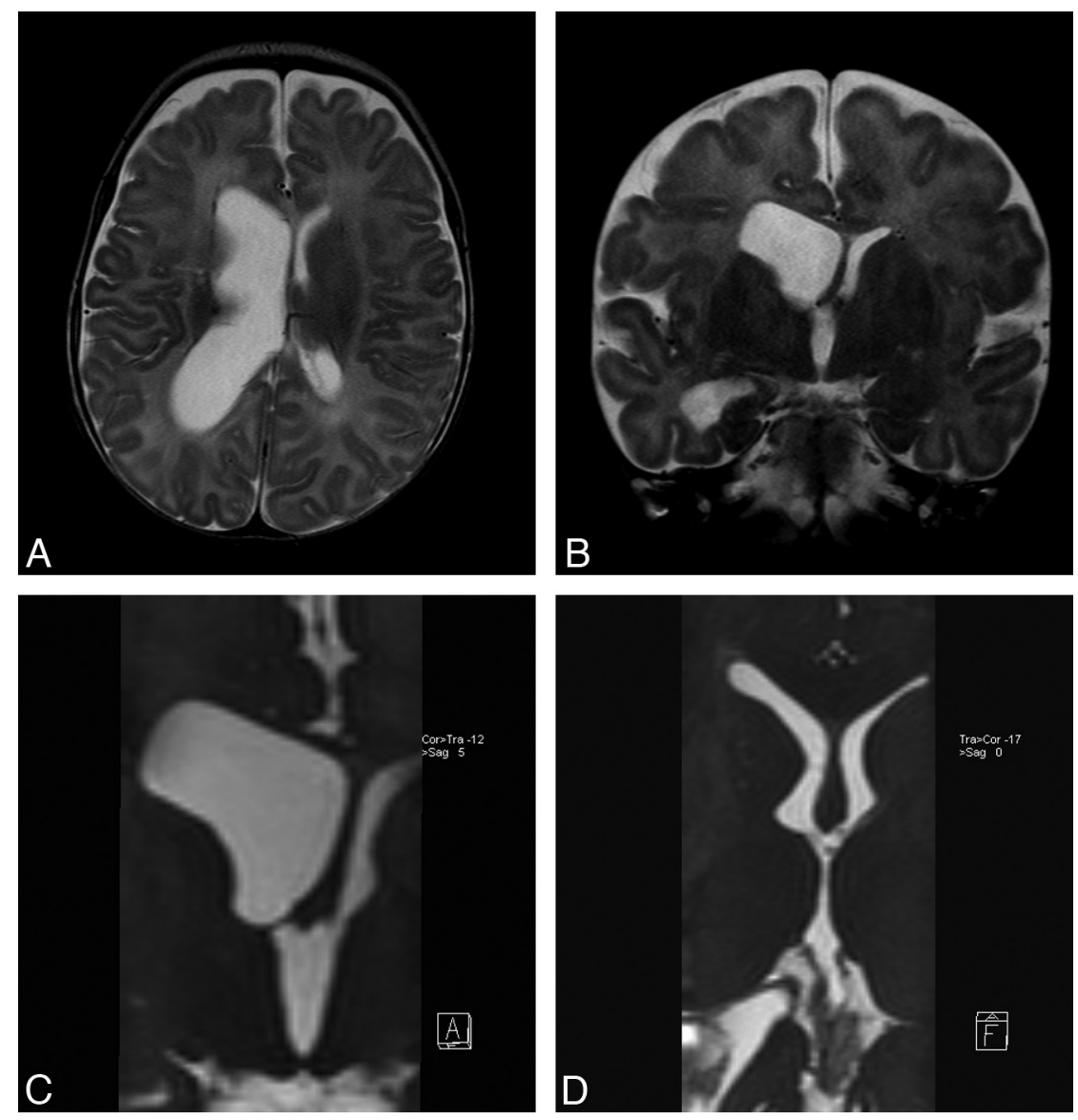

Fig 5. Case 4. A, Axial TSE T2 image through the lateral ventricle demonstrates unilateral right lateral ventriculomegaly. $B$, Coronal TSE T2 image through the foramen of Monro falsely demonstrates a free communication between the right lateral ventricle and the third ventricle. $C$, Coronal reformatted sagittal 3D-CISS image points out a complete membranous obstruction in the right foramen of Monro. D, Follow-up axial-oblique reformatted image revealing complete removal of obstructing membrane and normal-appearing lateral ventricles.

clearly demonstrated a membrane obstructing the right foramen of Monro completely (Fig 5C). Endoscopic fenestration was performed, and axial-oblique reformatted images with 3D-CISS sequence demonstrated free continuation between the lateral and third ventricle through the normal-appearing foramen of Monro at 6-month follow-up (Fig 5D).

\section{Discussion}

In our prospective study cohort, membranous obstruction was demonstrated in 92 (68.6\%) of 134 cases of hydrocephalus with the use of 3D-CISS at 3T. Intraventricular membranous obstruction is the most common pathologic finding to cause noncommunicating hydrocephalus (85/114 total noncommunicating cases). However, except in the cerebral aqueduct region, conventional images are insensitive in detection of obstructive membranes in the CSF pathway. In this study, they were only demonstrated in 57 (36.3\%) of 157 obstruction sites with the use of conventional imaging. In choosing the most appropriate treatment technique and to assess the prognosis in hydrocephalus, the classification of hydrocephalus and demonstration of obstruction, if it exists, are of significant importance. ${ }^{20,33}$ The terminology and the classification of hydrocephalus are still a matter of debate and are not the aims of our study. However, it is obvious that we need accurate neuroimaging techniques to demonstrate CSF pathways precisely. Invasive cisternographic methods have been described and used, such as radionuclide cisternography, CT cisternography with iodinated contrast or air, and MR cisternography with gadolinium-based contrast. However, because of their mainly invasive nature, these methods have not gained widespread acceptance. $^{23,34,35}$

Although conventional T1-weighted and T2-weighted images have been used for diagnosis and classification of hydrocephalus, it is a far from ideal neuroimaging technique despite many improvements, such as turbo or fast spin-echo sequence, phased array coil technology, parallel imaging techniques, and flow compensation methods eliminating CSF and vascular flow artifacts. The disadvantages of conventional images when investigating cisternal anatomy are poor spatial resolution, poor contrast-to-noise ratio between the cisterns and neighboring structures, and CSF flow effects when looking at CSF spaces. On the other hand, although cine PC is capable of showing flow as well as quantifying it, there are several disadvantages. ${ }^{36}$ Cine PC demonstrates only bidirectional flow in a selected direction (eg, craniocaudal or caudocranial flow in the sagittal plane). It is possible to obtain multiple cine PC sequences in different anatomic locations, though not practical. Whereas cine PC shows CSF flow, it is unable to demonstrate cisternal anatomic details.

The 3D-CISS sequence preoperatively facilitated selection of neuroendoscopy in the treatment of another 26 (19.4\%) of 134 patients who would have otherwise been treated with 
shunt insertion. Adding the cisternal obstruction-only cases, which were visible only on 3D-CISS and were treated with neuroendoscopy (total 5 cases), the total increased to 31 cases, further reducing the number of shunts inserted in our unit. Another advantage of 3D-CISS has been not only demonstrating the membranes within the prepontine cistern, but also guiding the procedure, showing the location, number, and the extent of the membranes. Therefore, ETV was not performed in 2 cases, with only the cisternal membrane that had extended caudally with widespread distribution on the basis of 3D-CISS findings. Fenestration of these membranes is crucial for success of the procedure. This finding was similar to that of Laitt et al. ${ }^{12}$ Laitt et al described the use of 3D-CISS sequence in 42 patients with hydrocephalus and demonstrated the value of this technique in various patient populations, including those with inflammatory or posthemorrhagic hydrocephalus. In their study, intraventricular cysts were demonstrated on 3DCISS images, which were not seen on conventional T1weighted and T2-weighted images. ${ }^{12}$ In this series, 5 of 7 patients with acute shunt malfunction who were investigated with 3D-CISS sequence were treated with endoscopic fenestration and became shunt free. The authors hence concluded that patients with communicating hydrocephalus secondary to inflammation or hemorrhage have an obstructive component that may benefit from endoscopic division of these membranes.

Usefulness of the 3D-CISS images has been shown in detailing the microsurgical anatomy in the brain, which aids surgical planning and approaches, ${ }^{3,5-8,10,11}$ as well as in evaluation of other neurosurgical pathologic findings such as hypothalamic hamartomas in which high CSF/tissue contrast is required. ${ }^{9}$ 3D-CISS images, originally developed for MR cisternography, are strongly T2/T1-weighted with extremely high spatial resolution and CSF/brain tissue contrast. ${ }^{12,13}$ The 3DCISS sequence also avoids the motion-induced signal intensity reduction of CSF with use of symmetric flow-compensating gradient pulses. ${ }^{12}$ Because of these special features of the 3DCISS sequence, we have been able to demonstrate the site of obstruction and the thin membranous structures at various parts of the CSF pathway.

A disadvantage of the 3D-CISS sequence may be its poor tissue distinction within the brain parenchyma. However, this technique is aimed at tissue/fluid distinction for which it is highly sensitive and specific. Another disadvantage of this sequence could be banding artifacts. This finding is seen rarely and to make a distinction between banding artifacts and real membrane is relatively easy because of the characteristic shape and periodicity of the artifacts. Lowering the flip angle to $50^{\circ}$ instead of $70^{\circ}$ helps to eliminate aliasing artifacts and decrease the specific absorption rate at $3 \mathrm{~T} .{ }^{37,38}$ Furthermore, because of relatively long scanning time, motion artifacts could be problematic; however, when performing the examination under sedation for patients younger than 10 years, we have rarely seen motion artifacts. Although increased signal-to-noise ratio and contrast-to-noise ratio at $3 \mathrm{~T}$ could influence the demonstration of membranes, the contribution of $3 \mathrm{~T}$ instead of $1.5 \mathrm{~T}$ imaging on demonstration of numerous sites of membranous obstruction in this study cohort is not obvious. Unfortunately, we did not perform any comparative study between $1.5 \mathrm{~T}$ and $3 \mathrm{~T}$.
To our knowledge, the current series is the largest prospective study illustrating the value of $3 \mathrm{D}$-CISS in the visualization and diagnosis of thin membranes obstructing CSF pathways. Furthermore, this is the first 3T study on hydrocephalus, including the 3D-CISS sequence. Identification of the nature and site of the obstruction, as well as distribution, significantly influences the selection of the appropriate management strategy by the neurosurgeon because the endoscopic techniques can be used to avoid permanent shunt insertion. The decision to insert a CSF shunt for hydrocephalus is one that is not taken lightly by neurosurgeons. Complications of CSF shunt implantation are many and are often significant. ${ }^{39}$ Approximately $50 \%$ of subjects younger than 2 years and $30 \%$ of those older than 2 years go on to have complications, including mechanical malfunctions, followed by infection, overdrainage, and ventricular septation. ${ }^{39}$ Shunt complications are also found to be responsible for most of the shunt-related deaths. ${ }^{40,41}$ On the other hand, the newer endoscopic procedures are found to have significantly less long-term complications, in particular, no risk for mechanical failure and significantly lower infection risk ( $1 \%-5 \%$ vs $1 \%-20 \%$ with shunts), whereas the infection in these cases runs a benign course. ${ }^{39}$ Furthermore, endoscopic management of intraventricular CSF cysts is shown to be effective and safe. ${ }^{42}$ Detailed knowledge of intraventricular and foraminal anatomy, which could be supported precisely by 3D-CISS, is crucial because anatomic hitches (eg, intraventricular septa, small foramen of Monro, or thick ventricular floor) are responsible for interruption of endoscopic procedures, intraoperatively in many cases. $^{12,43}$

Awaji et $\mathrm{al}^{16}$ recently compared intraoperative endoscopic findings with imaging by using a 3D-CISS sequence in 4 patients with arachnoid cysts. They found exact correlation of CISS imaging and anatomic structures during endoscopic procedure, whereas in only 1 case the $\mathrm{T} 2$ image corresponded to the intraoperative findings. Previously, Carpentier et $\mathrm{al}^{44}$ described functional fourth ventricular obstruction by partially fenestrated foramina of Luschka and Magendie by their alignment against the dura. Our findings were similar to 4 cases reported by Kurihara et $\mathrm{al}^{13}{ }^{13}$ in which thin membranes were demonstrated only on 3D-CISS sequence at the foramen of Monro and superior medullary velum. Aleman et $\mathrm{al}^{15} \mathrm{re-}$ ported 14 cases of hydrocephalus investigated with 3D-CISS sequence, all of which were treated with neuroendoscopic procedures. They found 3D-CISS sequence to be superior to conventional MR imaging in the demonstration of thin membranes and it allowed better understanding of the cause of hydrocephalus as well as postoperative evaluation. In an earlier, similar study, Alemen et $\mathrm{al}^{15}$ demonstrated intraventricular septa in 11 of 15 neuroendoscopic cases not demonstrated on T2-weighted images, thus showing the potential cause of hydrocephalus.

\section{Conclusions}

In this large study cohort, we have further demonstrated the value of 3D-CISS sequence to establish the potential cause in patients with hydrocephalus, identifying patients with noncommunicating hydrocephalus in $26(22.8 \%)$ of 114 of our patients initially diagnosed with communicating hydrocephalus. Furthermore, this technique confirmed the site of ob- 
struction in 31 cases that were appropriate for neuroendoscopic intervention and avoiding insertion of shunts with their significant associated morbidity and mortality rates. On the basis of these findings, we recommend 3D-CISS sequence as the investigation of choice in all cases of hydrocephalus.

\section{References}

1. Casselman JW, Kuhweide R, Deimling M, et al. Constructive interference in steady state-3DFT MR imaging of the inner ear and cerebellopontine angle. AJNR Am J Neuroradiol 1993;14:47-57

2. Fushimi Y, Miki Y, Ueba T, et al. Liliequist membrane: three-dimensional constructive interference in steady state MR imaging. Radiology 2003;229: $360-65$

3. Kakizawa Y, Hongo K, Takasawa H, et al. "Real" three-dimensional constructive interference in steady-state imaging to discern microneurosurgical anatomy. Technical note. J Neurosurg 2003;98:625-30

4. Lane JI, Ward H, Witte RJ, et al. 3-T imaging of the cochlear nerve and labyrinth in cochlear-implant candidates: $3 \mathrm{D}$ fast recovery fast spin-echo versus $3 \mathrm{D}$ constructive interference in the steady state techniques. AJNR Am J Neuroradiol 2004;25:618-22

5. Yousry I, Moriggl B, Schmid UD, et al. Trigeminal ganglion and its divisions: detailed anatomic MR imaging with contrast-enhanced 3D constructive interference in the steady state sequences. AJNR Am J Neuroradiol 2005;26: $1128-35$

6. Yousry I, Camelio S, Schmid UD, et al. Visualization of cranial nerves I-XII: value of 3D CISS and T2-weighted FSE sequences. Eur Radiol 2000;10:1061-67

7. Yagi A, Sato N, Taketomi A, et al. Normal cranial nerves in the cavernous sinuses: contrast-enhanced three-dimensional constructive interference in the steady state MR imaging. AJNR Am J Neuroradiol 2005;26:946-50

8. Hirai T, Kai Y, Morioka M, et al. Differentiation between paraclinoid and cavernous sinus aneurysms with contrast-enhanced $3 \mathrm{D}$ constructive interference in steady- state MR imaging. AJNR Am J Neuroradiol 2008;29:130-33

9. Yamura M, Hirai T, Korogi Y, et al. Evaluation of small hypothalamic hamartomas with 3D constructive interference in steady state (CISS) sequence. $\mathrm{Neu}$ roradiology 2005;47:204-08

10. Everton KL, Rassner UA, Osborn AG, et al. The oculomotor cistern: anatomy and high-resolution imaging. AJNR Am J Neuroradiol 2008;29:1344-48

11. Davagnanam I, Chavda SV. Identification of the normal jugular foramen and lower cranial nerve anatomy: contrast-enhanced 3D fast imaging employing steady-state acquisition MR imaging. AJNR Am J Neuroradiol 2008;29:574-76

12. Laitt RD, Mallucci CL, Jaspan T, et al. Constructive interference in steady-state 3D Fourier-transform MRI in the management of hydrocephalus and third ventriculostomy. Neuroradiology 1999;41:117-23

13. Kurihara N, Takahashi S, Tamura $\mathrm{H}$, et al. Investigation of hydrocephalus with three-dimensional constructive interference in steady state MRI. Neuroradiology 2000;42:634-38

14. Doll A, Christmann D, Kehrli P, et al. Contribution of 3D CISS MRI for preand post-therapeutic monitoring of obstructive hydrocephalus. J Neuroradiol 2000;27:218-25

15. Aleman J, Jokura H, Higano S, et al. Value of constructive interference in steady-state three-dimensional, Fourier transformation magnetic resonance imaging for the neuroendoscopic treatment of hydrocephalus and intracranial cysts. Neurosurgery 2001;48:1291-95

16. Awaji M, Okamoto K, Nishiyama K. Magnetic resonance cisternography for preoperative evaluation of arachnoid cysts. Neuroradiology 2007;49:721-26

17. Kunz M, Schulte-Altedorneburg G, Uhl E, et al. Three-dimensional constructive interference in steady-state magnetic resonance imaging in obstructive hydrocephalus: relevance for endoscopic third ventriculostomy and clinical results. J Neurosurg 2008;109:931-38

18. Lü J, Zhu XL. Cranial arachnoid membranes: some aspects of microsurgical anatomy. Clin Anat 2007;20:502-11
19. Fushimi Y, Miki Y, Takahashi JA, et al. MR imaging of Liliequist's membrane. Radiat Med 2006;24:85-90

20. Mori K. Hydrocephalus-revision of its definition and classification with special reference to "intractable infantile hydrocephalus". Childs Nerv Syst 1990;6:198-204

21. Oi S, Di Rocco C. Proposal of "evolution theory in cerebrospinal fluid dynamics" and minor pathway hydrocephalus in developing immature brain. Childs Nerv Syst 2006;22:662-69

22. Hoare RD, Strand RD. The third ventricle in hydrocephalus due to basal cistern block. Pediatr Radiol 1974;2:15-21

23. Yamada H, Nakamura S, Tanaka Y, et al. Ventriculography and cisternography with water-soluble contrast media in infants with myelomeningocele. Radiology 1982;143:75-83

24. Kehler U, Gliemroth J. Extraventricular intracisternal obstructive hydrocephalus-a hypothesis to explain successful 3rd ventriculostomy in communicating hydrocephalus. Pediatr Neurosurg 2003;38:98-101

25. Longatti P, Fiorindi A, Feletti A, et al. Endoscopic opening of the foramen of magendie using transaqueductal navigation for membrane obstruction of the fourth ventricle outlets. Technical note. J Neurosurg 2006;105:924-27

26. Inamura $T$, Morioka $T$, Nishio $S$, et al. Diverticular enlargement of the foramina of Luschka and congenital hydrocephalus. Childs Nerv Syst 2002;18:652-55

27. Huang YC, Chang CN, Chuang HL, et al. Membranous obstruction of the fourth ventricle outlet. A case report. Pediatr Neurosurg 2001;35:43-47

28. Rifkinson-Mann S, Sachdev VP, Huang YP. Congenital fourth ventricular midline outlet obstruction. Report of two cases. J Neurosurg 1987;67:595-99

29. Rougier A, Ménégon P. MRI evidence of membranous occlusion of the foramen of Magendie. Acta Neurochir 2009;151:693-94. Epub 2009 Mar 5

30. Hashish H, Guenot M, Mertens P, et al. Chronic hydrocephalus in an adult due to congenital membranous occlusion of the apertura mediana ventriculi quartii (foramen of Magendie). Report of two cases and review of the literature. Neurochirurgie 1999;45:232-36

31. Caporal R, Segrestaa JM, Dorf G. Endocrine expressions of hydrocephalus. A case of primary amenorrhoea revealing a stenosis of the foramen of Magendie. Acta Endocrinol 1983;102:161-66

32. Lukin RR, Chambers AA, Soleimanpour M. Outlet obstruction of the fourth ventricle in sarcoidosis. Neuroradiology 1975;10:65-68

33. Beni-Adani L, Biani N, Ben-Sirah L, et al. The occurrence of obstructive vs absorptive hydrocephalus in newborns and infants: relevance to treatment choices. Childs Nerv Syst 2006;22:1543-63

34. Enzmann DR, Norman D, Price DC, et al. Metrizamide and radionuclide cisternography in communicating hydrocephalus. Radiology 1979;130:681-86

35. Joseph VB, Raghuram L, Korah IP, et al. MR ventriculography for the study of CSF flow. AJNR Am J Neuroradiol 2003;24:373-81

36. Quencer RM. Intracranial CSF flow in pediatric hydrocephalus: evaluation with cine-MR imaging. AJNR Am J Neuroradiol 1992;13:601-08

37. Wu ML, Ko CW, Chen TY, et al. MR ventriculocisternography by using $3 \mathrm{D}$ balanced steady-state free precession imaging: technical note. AJNR Am J Neuroradiol 2005;26:1170-73

38. Scheffler K, Lehnhardt S. Principles and applications of balanced SSFP techniques. Eur Radiol 2003;13:2409-18

39. Di Rocco C, Massimi L, Tamburrini G. Shunts vs endoscopic third ventriculostomy in infants: are there different types and/or rates of complications?. Childs Nerv Syst 2006;22:1573-89

40. Bryant MJ, McEniery J, Walker DG, et al. Preliminary study of shunt related death in paediatric patients. J Clin Neurosci 2004;11:614-15

41. Tuli S, Tuli J, Drake J, et al. Predictors of death in pediatric patients requiring cerebrospinal fluid shunts. J Neurosurg 2004;100(5 Suppl Pediatrics):442-46

42. Tamburrini G, D'Angelo L, Paternoster G, et al. Endoscopic management of intra and paraventricular CSF cysts. Childs Nerv Syst 2007;23:645-51

43. Jones RF, Kwok BC, Stening WA, et al. The current status of endoscopic third ventriculostomy in the management of non-communicating hydrocephalus. Minim Invasive Neurosurg 1994;37:28-33

44. Carpentier A, Brunelle F, Philippon J, et al. Obstruction of Magendie's and Luschka's foramina. Cine-MRI, aetiology and pathogenesis. Acta Neurochir (Wien) 2001;143:517-21 